\title{
DIVERSIDADE E ESTRUTURA GENÉTICA ESPACIAL DE Calophyllum brasiliense CAMB. (CLUSIACEAE) EM UMA FLORESTA PALUDOSA ${ }^{1}$
}

\author{
Cristiane Aparecida Fioravante Reis ${ }^{2}$, Anderson Marcos de Souza ${ }^{3}$, Evânia Galvão Mendonça ${ }^{4}$, Flávio \\ Rodrigues Gonçalvez ${ }^{5}$, Rodrigo Magno Guimarães Melo ${ }^{6}$ Dulcinéia de Carvalho ${ }^{7}$
}

\begin{abstract}
RESUMO - As áreas de ocorrência de florestas paludosas se encontram alteradas e restritas devido aos processos de destruição e fragmentação, com consequente redução do seu tamanho populacional. Calophyllum brasiliense Camb. é uma espécie arbórea abundante em ambientes ciliares, devido à sua preferência em colonizar solos com alta saturação hídrica, sendo considerada especialista em hábitat. Além das consequências ecológicas, como mortalidade e baixo recrutamento, poderá ocorrer perda da variabilidade genética, comprometendo a viabilidade da espécie no local. Nesse contexto, foi realizado um censo da espécie em um fragmento de floresta paludosa, sendo estabelecidas quatro classes de altura para a análise genética. Os marcadores aloenzimáticos revelaram 11 locos polimórficos, com um número médio de 2,0 alelos em cada loco, não sendo observada a perda ou a fixação de alelos e polimorfismo de $100 \%$. As Classes I e III apresentaram excesso de homozigotos, sendo esse valor não significativo para na Classe I. A heterozigosidade foi superior à esperada nas Classes II e IV. Os valores de diversidade genética encontrados na espécie estudada foram considerados altos em relação aos valores relatados em outras espécies arbóreas. Portanto, a ocorrência de mutações e a incorporação de novos alelos na população são elevadas, além de aumentarem o número de recombinações e múltiplas paternidades nas progênies. No geral, a análise da distribuição espacial dos genótipos foi aleatória nas classes analisadas e, na Classe I, os indivíduos próximos a uma distância de $10 \mathrm{~m}$ apresentaram estrutura de família. A alta diversidade genética da espécie e a ausência de estruturação espacial dos genótipos na maioria das classes analisadas devem ser consideradas em planos de conservação dessa área.
\end{abstract}

Palavras-chave: Aloenzimas, cohorts e estrutura temporal.

\section{GENETIC DIVERSITY AND SPATIAL GENETIC STRUCTURE OF Calophyllum brasiliense CAMB. (CLUSIACEAE) IN A SWAMPY FOREST}

\begin{abstract}
The areas of occurrence of swampy forests have been altered and restricted due to destruction and fragmentation processes, with consequent reduction of their population size. Calophyllum brasiliense Camb. is an abundant tree species in Riparian environments for its preference for colonizing high water saturation soils, being considered specialist in habitat. Besides the ecological consequences, such as mortality and low recruitment, genetic variability loss can occur, compromising the viability of the species in the place. Thus, molecular markers are necessary to evaluate the genetic effects. Within this context, a census of the species was conducted in a swampy forest fragment, with four height classes being established for genetic analysis. The alloenzymatic markers revealed a total of 11 polymorphic loci with a mean number of 2.0 alleles in each locus it was not observed the loss or alleles fixation. The observed heterozigosity was larger than that
\end{abstract}

\footnotetext{
${ }^{1}$ Recebido em 17.09.2007 e aceito para publicação em 06.03.2009.

${ }^{2}$ ProgramadePós-Graduaçãoem GenéticaeMelhoramentodePlantasdaUniversidadeFederal deLavras(UFLA).E-mail: <cristianemep@yahoo.com.br>.

${ }^{3}$ Departamento de Engenharia Florestal da Universidade Federal do Piauí (UFPI). E-mail: <andermsouza@ yahoo.com.br>.

${ }^{4}$ Programa de Pós-Graduação em Biotecnologia da UFLA. E-mail: <evaniafloresta@ hotmail.com>.

${ }^{5}$ Programa de Pós-Graduação em Ciências Florestais da UFLA. E-mail: <flavio@ katatudo.com.br>.

${ }^{6}$ Biólogo formado na Escola Superior em Meio Ambiente ESMA/Iguatama-MG.

${ }^{6}$ Departamento de Ciências Florestais da UFLA. E-mail: <dulce@ ufla.br>.
} 
expected for Classes II and IV, evidencing heterozygote excess. Classes I and III presented homozygote excess, the value being non significant for Class I. The genetic diversity values found were considered high compared to other tree species. Thus, the occurrence of mutations and the incorporation of new alleles in the population are high, besides increasing the recombination number and multiple paternities in the progenies. Overall, the analysis of the spatial distribution of the genotypes was random for most classes analyzed, except for Class I, where the individuals close to a $10 \mathrm{~m}$ distance presented family structure. The high genetic diversity of this species and absence of spatial structuring of the genotypes in most of the classes analyzed should be considered for conservation planning of this area.

Keywords: Isoenzyme, cohorts and temporal structure.

\section{INTRODUÇÃO}

Das fitofisionomias remanescentes da Floresta Atlântica no Estado de Minas Gerais, as formações ciliares são as mais sujeitas a perturbações, principalmente pela presença de solos relativamente férteis e úmidos, atributos ideais para a agricultura e formação de pastagem e, também pela alta diversidade de espécies, sendo estas fonte de madeira e de produtos florestais madeiráveis e não-madeiráveis. Dentre as formações ciliares, as florestas paludosas ou florestas de brejo, também denominadas de florestas latifoliadas higrófilas, são florestas estabelecidas sobre solos hidromórficos, com presença quase permanente de água na superfície do solo em função do afloramento do lençol freático (TORRES et al., 1994; IVANAUSKAS et al., 1997). As formações ciliares ou ripárias apresentam funções chave na regulação de habitats, promovendo a manutenção da qualidade e quantidade de água, retendo grande quantidade de nutrientes e defensivos agrícolas, protegendo o solo dos efeitos erosivos. Além disso, possuem atrativos ideais para o abrigo e alimentação da fauna silvestre e atuam como corredores ecológicos, permitindo a ocorrência de fluxo gênico efetivo de espécies da fauna e da flora, sendo de grande importância para a conservação da biodiversidade.

Calophyllum brasiliense Camb. é uma espécie arbórea característica e exclusiva das florestas pluviais localizadas sobre solos úmidos e brejosos, sendo capaz de crescer dentro d'água e até em áreas de mangue (LORENZI, 1992). A condição hídrica local é um dos fatores que influenciam a sua distribuição e a sua capacidade em se desenvolver em locais com saturação hídrica faz com esta espécie tenha vantagens sobre outras (FISHER, 1990; SCHIAVINI, 1992; MARQUES, 1994; KAWAGUICI, 1994). Taxonomicamente pertencente à família Clusiaceae (ex Guttiferae), C. brasiliense é uma espécie conhecida popularmente em todo Brasil como guanandi. Ocorre em todas as bacias hidrográficas brasileiras, sendo comum desde a latitude $18^{\circ} \mathrm{N} \mathrm{em}$ Porto Rico até $28^{\circ} 10^{\prime} \mathrm{S}$ em Santa Catarina. Apresenta flores reunidas em curtos racemos axilares, ordenadas em címulos trifloros, brancas e de duas formas: masculinas (com muitos estames) e hermafroditas ou bissexuais (com poucos estames), reunidas na mesma árvore. Ecologicamente é considerada uma espécie clímax e a dispersão de suas sementes pode ser por morcegos frugívoros (quiroptecórica), aves (ornitocórica) como a gralha-azul (Cyanocorax caeruleus - Corvidade, no sul do Brasil) ou hidrocórica, devido a sua ocorrência frequente em cursos d'água (CARVALHO, 1994).

O estabelecimento de um programa de conservação in situ de populações arbóreas requer o conhecimento de padrões ecológicos e genéticos. Assim, a utilização de marcadores moleculares, tem permitido um grande avanço nos estudos de genética populacional de espécies arbóreas florestais, principalmente para descrever a organização da variação genética em populações naturais de plantas (HAMRICK et al., 1981; LOVELESS e HAMRICK, 1984; HAMRICK e LOVELESS, 1986; BOTRELeCARVALHO, 2004; PINTOetal., 2004; VIEIRA e CARVALHO, 2008). A variabilidade é introduzida continuamente nas populações por mutação ou migração de indivíduos de outras populações e, pode ser perdida por deriva genética, endocruzamentos e no caso de genes neutros, pela seleção natural (COLE, 2003). A existência de variação genética é um fator fundamental para que ocorra a evolução. A seleção natural atua entre as variantes dentro das populações em relação à adaptação ao ambiente, proporcionando variação entre as mesmas e, por fim, variação entre espécies (TORGGLER et al., 1995). Dessa forma, uma população com elevada variabilidade genética, aliada a fatores demográficos e ecológicos, teria maior chance de 
perpetuação. A manutenção da variabilidade genética em populações é base para a conservação de espécies (YEEH et al., 1996) e, portanto, a sua descrição e distribuição são fundamentais para o estabelecimento de práticas conservacionistas efetivas. Os objetivos deste trabalho foram caracterizar os padrões da variabilidade genética de $C$. brasiliense, em um remanescente de floresta paludosa, quantificando os níveis de diversidade genética em diferentes classes de altura, representativas de fases ontogênicas (plântulas, árvores jovens e adultas) e, conhecer a distribuição espacial dos genótipos, de forma a propor estratégias para a conservação desta espécie no local estudado.

\section{MATERIAL E MÉTODOS}

\subsection{Caracterização da área de estudo}

A área de estudo localiza-se no Sul do Estado de Minas Gerais a aproximadamente $6 \mathrm{~km}$ do município de Lavras, na encosta da Serra do Carrapato. A vegetação predominante pode ser definida como uma disjunção do bioma Cerrado, inserida dentro da área de distribuição das Florestas Estacionais Semideciduais do Sudeste brasileiro (CASTRO, 2004; IBGE, 1993). Os solos dominantes são Latossolos Vermelho-Amarelos e Vermelho distróficos e Cambissolos Háplicos Tb distróficos (CASTRO, 2004). O clima da região, segundo a classificação de Köppen, é do tipo Cwb, com precipitação média anual de 1493,2 mm e temperatura média anual de $19,3{ }^{\circ} \mathrm{C}$ (MACHADO et al., 2004).

Segundo Vieira e Carvalho (2008), na paisagem atual da área há um número reduzido de pequenos remanescentes florestais; uma área matricial (circundante das manchas de vegetação), de baixa biomassa, composta por lavouras de café e pastagens plantadas (Brachiaria spp.) para a criação de gado; além de corredores de vegetação. $\mathrm{O}$ fragmento em estudo apresenta evidência de impactos localizados no seu interior, causados principalmente pela constante penetração de gado, formando trilhas e prejudicando a regeneração em vários trechos, porém não foram observados sinais evidentes de exploração seletiva de madeira. A presença de solo permanentemente encharcado, com fluxo constante ou temporário de água superficial dentro de pequenos canais é comum no interior do fragmento e, pode estar associada ao grau de preservação e também aos efeitos da antropização sobre a matriz vegetacional.

\subsection{Amostragem}

A área de estudo compreende um remanescente de Floresta Semidecidual, de 1,0 ha, sendo que a área de floresta paludosa possui $2.600 \mathrm{~m}^{2}$, onde foram alocadas 24 parcelas de $10 \times 10$ m. Para avaliar a distribuição das plântulas, árvores jovens e adultas de C. brasiliense foi realizado um censo e as coordenadas $\mathrm{x}$ e y de todos os indivíduos foram anotadas para se obter a sua posição dentro das parcelas e posterior análise da distribuição espacial dos genótipos. Em todas as parcelas foram medidas as alturas dos indivíduos, ficando estabelecida como Classe I, os indivíduos com altura entre 0,3 e $1 \mathrm{~m}$; Classe II, os indivíduos com altura entre 1,01 e 3,0 m; Classe III, com alturas variando entre 3,01 e 9,0 $\mathrm{m}$ e Classe IV, os indivíduos acima de 9,0 m. Os indivíduos até $3 \mathrm{~m}$ de altura foram classificados como plântulas (Classes I e II), aqueles com alturas de 3 a $9 \mathrm{~m}$ como jovens (Classe III) e aqueles com alturas superiores a $9 \mathrm{~m}$ como adultos (Classe IV). Folhas foram coletadas para a extração das enzimas em todos os indivíduos amostrados, em cada classe de altura. O material coletado em campo foi acondicionado em saco plástico, identificado e armazenado em caixas de isopor com gelo. No Laboratório de Conservação Genética de Espécies Arbóreas do Departamento de Ciências Florestais da UFLA, as folhas foram armazenadas a $-80^{\circ} \mathrm{C}$ até o momento da extração.

\subsection{Extração das enzimas e eletroforese}

No procedimento de extração das enzimas foram usados $300 \mathrm{mg}$ de tecido do limbo foliar, $5 \mathrm{mg}$ de Polivinilpirrolidona - PVP e $1 \mathrm{~mL}$ de solução tampão $\mathrm{n}^{\circ} 1$ de Alfenas et al. (1998). O tecido foliar foi macerado utilizando pistilo e almofariz de porcelana, previamente resfriados e, os extratos obtidos na maceração foram armazenados em tubos de 1,5 mL, previamente identificados e armazenados $\mathrm{a}-80^{\circ} \mathrm{C}$. Para a eletroforese foi utilizada cuba vertical, tendo como meio suporte o gel de poliacrilamida ( $4 \%$ para o gel de concentração e 7,5\% para o de separação), onde foram aplicados $30 \mu \mathrm{L}$ de sobrenadante dos extratos de cada amostra. Durante a eletroforese foi utilizada amperagem de 10 $\mathrm{mA}$ por gel, 300 Volts, à temperatura de $4^{\circ} \mathrm{C}$, tendo a corrida eletroforética duração de 3 horas e 30 minutos aproximadamente. Os procedimentos de preparo do gel, aplicação das amostras, eletroforese e revelação dos géis seguiram a metodologia descrita por Alfenas et al. (1998).

R. Árvore, Viçosa-MG, v.33, n.2, p.265-275, 2009 
Nove sistemas enzimáticos foram testados para a caracterização da variabilidade genética, em função de apresentarem boa resolução do padrão de bandas e polimorfismo dos locos, sendo eles: Álcool desidrogenase $(\mathrm{ADH}), \alpha$-Esterase $(\alpha$-EST), $\beta$-Esterase $(\beta$-EST), Fosfatase ácida (ACP), Glucose desidrogenase (GDH), Glutamato desidrogenase (GTDH), Malato desidrogenase (MDH), Sorbitol desidrogenase (SDH) e Xiquimato desidrogenase (SKDH). Após o aparecimento das bandas, os géis foram retirados da solução de revelação, lavados em água corrente e a leitura das bandas nos géis foi realizada sobre a superfície de um diafanoscópio. A identificação dos locos e dos alelos foi feita a partir da região mais catódica para a mais anódica. As identificações e as interpretações dos zimogramas foram realizadas seguindo a mesma definição dos padrões de enzimas disponíveis na literatura (ALFENAS et al., 1991).

\subsection{Caracterização da variabilidade genética e da distribuição espacial dos genótipos}

A caracterização da variabilidade genética presente nos indivíduos de $C$. brasiliense foi realizada a partir da determinação dos genótipos de cada indivíduo, sendo estes dados analisados por meio dos programas computacionais Genetic Data Analysis-GDA (LEWIS e ZAYKIN, 2000) e BIOSYS 1 (SWOFFORD e SELANDER, 1989).

Para análise da estrutura espacial de $C$. brasiliense foi utilizado o programa SPAGeDi versão 1.1 (HARDY e VEKEMANS, 2002). O coeficiente de coancestria com base em Ritland (1996) foi estimado entre plantas para cada uma das classes de distância, para o número total de plantas e para o conjunto dos dados das Classes III e IV, de menor número amostral. O erro padrão da média das estimativas foi obtido por reamostragem jackknife entre locos e, a partir dele, foram construídos intervalos de confiança a $95 \%$ de probabilidade do coeficiente de coancestria médio estimado para cada classe de distância. A ausência de estrutura genética espacial foi testada dentro de cada classe de distância utilizando-se 1.000 permutações.

\section{RESULTADOS E DISCUSSÃO}

\subsection{Número de indivíduos amostrados}

O censo realizado possibilitou a identificação de 746 indivíduos de C. brasiliense. O número de indivíduos por classe de altura e o número de amostras por classe utilizadas para a análise aloenzimática se encontram na Tabela 1. A diminuição do número de indivíduos da Classe I (plântula) para a Classe IV (adulta) é relatada em vários trabalhos (CHUNG et al., 2003; CONTE et al., 2003; RUSCHEL et al., 2006; VIEIRA et al., 2008).

\subsection{Sistemas enzimáticos}

Para a caracterização da variabilidade genética de $C$. brasiliense, os nove sistemas enzimáticos testados apresentaram resolução do padrão de bandas e polimorfismo dos locos adequados, em todas as quatro classes de altura analisadas. Nestes sistemas, 11 locos se mostraram passíveis de interpretação, apresentando estrutura monomérica da enzima e com segregação de dois alelos em cada loco. Os sistemas ACP, ADH, GDH, GTDH, MDH, SDH e SKDH apresentaram apenas um loco passível de interpretação, na região mais catódica. Nos sistemas $\alpha$-EST e $\beta$-EST foi possível a interpretação de dois locos, um na região catódica e outro na anódica.

O número de locos polimórficos utilizados com marcadores aloenzimáticos em espécies arbóreas é bastante variável (VIEIRA e CARVALHO, 2008; JAEGER et al., 2007; MORAES et al., 2005; CARVALHO e OLIVEIRA, 2004). Ng et al. (2004) e Telles et al. (2003) consideraram a partir de quatro locos, um número considerável para se avaliar a variabilidade genética. Entretanto, Berg e Hamrick (1997) sugerem um número mínimo de 10 locos polimórficos para estudos de diversidade e estrutura genética. Portanto, o número de locos polimórficos obtidos para plântulas, árvores jovens e adultas de $C$. brasiliense neste estudo são suficientes para a avaliação e caracterização de sua variabilidade genética e distribuição espacial.

Tabela 1 - Classes de altura, número de indivíduos amostrados e número de indivíduos selecionados para a análise genética de uma população natural de Calophyllum brasiliense Camb., amostrada em um remanescente de floresta paludosa

Table 1 - Height classes, number of sampled individuals, and number of individuals selected for the genetic analysis of a natural population of Calophyllum brasiliense Camb., sampled in a swampy forest fragment

\begin{tabular}{lcc}
\hline Classes de Altura & Número de Indivíduos Amostrados & Número de Indivíduos Usados na Análise Genética \\
\hline I $(0,3$ a $1,0 \mathrm{~m})$ & 396 & 115 \\
II $(1,01$ a $3,0 \mathrm{~m})$ & 250 & 125 \\
III $(3,01$ a $9,0 \mathrm{~m})$ & 65 & 65 \\
IV (acima de $9,0 \mathrm{~m})$ & 35 & 35 \\
\hline Total & 746 & 340 \\
\hline
\end{tabular}

R. Árvore, Viçosa-MG, v.33, n.2, p.265-275, 2009 


\subsection{Frequências alélicas}

A análise das frequências alélicas das plântulas, árvores jovens e adultas de $C$. brasiliense, amostradas no fragmento de floresta paludosa, mostra segregação de dois alelos, não sendo detectadas a perda ou a fixação de alelos (Tabela 2). A distribuição das frequências alélicas apresenta pequenas variações dentro e entre as classes. As menores variações das frequências alélicas foram observadas na Classe I. Os maiores valores de frequências alélicas foram detectados no alelo 2 na maioria dos locos, exceto nos locos $\alpha$-EST1 da Classe I, ACP1 da Classe III e $\beta$-EST1 e ACP1 da Classe IV.

Tabela 2 - Frequências alélicas e tamanho da amostra (n) em 11 locos aloenzimáticos em uma população natural de Calophyllum brasiliense Camb.

Table 2 - Allelic frequencies and sample size ( $n$ ) in 11 alloenzymatic loci detected in a natural population of Calophyllum brasiliense Camb.

\begin{tabular}{|c|c|c|c|c|c|}
\hline \multirow[t]{2}{*}{$\overline{\text { Loco }}$} & \multirow[t]{2}{*}{ Alelos } & \multicolumn{4}{|c|}{ Classes de altura* } \\
\hline & & $\mathrm{I}$ & II & III & IV \\
\hline \multirow[t]{3}{*}{ ADH1 } & 1 & 0,421 & 0,256 & 0,269 & 0,333 \\
\hline & 2 & 0,579 & 0,744 & 0,731 & 0,667 \\
\hline & $\mathrm{n}$ & 107 & 123 & 65 & 33 \\
\hline \multirow[t]{3}{*}{$\overline{\alpha-E S T 1}$} & 1 & 0,531 & 0,376 & 0,397 & 0,464 \\
\hline & 2 & 0,469 & 0,624 & 0,603 & 0,536 \\
\hline & $\mathrm{n}$ & 98 & 89 & 58 & 28 \\
\hline \multirow[t]{3}{*}{$\overline{\alpha-E S T 2}$} & 1 & 0,477 & 0,403 & 0,465 & 0,414 \\
\hline & 2 & 0,523 & 0,597 & 0,535 & 0,586 \\
\hline & $\mathrm{n}$ & 109 & 108 & 57 & 29 \\
\hline \multirow[t]{3}{*}{$\overline{\beta-\mathrm{EST} 1}$} & 1 & 0,484 & 0,309 & 0,438 & 0,536 \\
\hline & 2 & 0,516 & 0,691 & 0,563 & 0,464 \\
\hline & $\mathrm{n}$ & 94 & 97 & 56 & 28 \\
\hline \multirow[t]{3}{*}{$\overline{\beta-E S T 2}$} & 1 & 0,487 & 0,394 & 0,469 & 0,394 \\
\hline & 2 & 0,513 & 0,606 & 0,531 & 0,606 \\
\hline & $\mathrm{n}$ & 113 & 118 & 65 & 33 \\
\hline \multirow[t]{3}{*}{$\overline{\mathrm{ACP} 1}$} & 1 & 0,478 & 0,360 & 0,780 & 0,757 \\
\hline & 2 & 0,522 & 0,640 & 0,220 & 0,243 \\
\hline & $\mathrm{n}$ & 115 & 114 & 65 & 35 \\
\hline \multirow[t]{3}{*}{$\overline{\mathrm{GDH} 1}$} & 1 & 0,306 & 0,269 & 0,235 & 0,200 \\
\hline & 2 & 0,694 & 0,731 & 0,765 & 0,800 \\
\hline & $\mathrm{n}$ & 111 & 121 & 65 & 35 \\
\hline \multirow[t]{3}{*}{$\overline{\text { GTDH1 }}$} & 1 & 0,214 & 0,310 & 0,205 & 0,206 \\
\hline & 2 & 0,786 & 0,690 & 0,795 & 0,794 \\
\hline & $\mathrm{n}$ & 103 & 121 & 65 & 34 \\
\hline \multirow[t]{3}{*}{$\overline{\mathrm{MDH} 1}$} & 1 & 0,279 & 0,302 & 0,226 & 0,200 \\
\hline & 2 & 0,721 & 0,698 & 0,774 & 0,800 \\
\hline & $\mathrm{n}$ & 102 & 124 & 62 & 35 \\
\hline \multirow[t]{3}{*}{$\overline{\mathrm{SDH} 1}$} & 1 & 0,290 & 0,252 & 0,203 & 0,271 \\
\hline & 2 & 0,710 & 0,748 & 0,797 & 0,729 \\
\hline & $\mathrm{n}$ & 112 & 125 & 64 & 35 \\
\hline \multirow[t]{3}{*}{$\overline{\mathrm{SKDH} 1}$} & 1 & 0,283 & 0,299 & 0,169 & 0,288 \\
\hline & 2 & 0,717 & 0,701 & 0,831 & 0,712 \\
\hline & $\mathrm{n}$ & 115 & 122 & 65 & 33 \\
\hline
\end{tabular}

* I $(0,3$ a $1,0 \mathrm{~m})$, II $(1,01$ a $3,0 \mathrm{~m})$, III $(3,01$ a $9,0 \mathrm{~m})$ e IV (acima de $9,0 \mathrm{~m}$ ).
O número de locos em equidade gênica foi igual a 54\% na Classe I, 36\% nas Classes II, III e IV. Segundo Frankel et al. (1996), a equidade gênica é conceituada como aquela na qual a menor variabilidade das frequências numa espécie indica maior diversidade genética. Essa maior diversidade mantém as populações menos susceptíveis à fixação e perda de alelos, quando submetidas à perturbações e gargalos genéticos. Portanto, uma população com maior diversidade genética estaria mais protegida dos efeitos da deriva do que aquelas com alelos em menor frequência, estando estes mais susceptíveis de serem perdidos no tempo. $\mathrm{Na}$ equidade gênica ou baixa amplitude de variação, consideram-se como similares dois alelos que apresentem frequências entre 0,350 e 0,650.

De acordo com Kawaguici e Kageyama (2001), a variação nas frequências alélicas entre as diferentes classes pode ter várias origens, tais como migração, mutação, seleção e deriva genética. Essas variações nas frequências alélicas, supostamente causadas pela deriva genética, foram observadas somente no loco ACP1, $\alpha$-EST2, $\beta$-EST2 e SKDH1. Por exemplo, o alelo 1 desse loco, apresenta maior frequência da Classe I $(0,478)$ em relação a Classe II $(0,360)$, menor frequência na II $(0,360)$ em relação a III $(0,780)$ e frequências próximas na Classe III $(0,780)$ e IV $(0,757)$.

\section{4. Índices de diversidade genética}

A partir da análise das frequências alélicas das plântulas, árvores jovens e adultas de C. brasiliense foram estimados os índices de diversidade genética (Tabela 3). O número médio de alelos por loco foi igual a 2,0 e o polimorfismo foi $100 \%$. As heterozigosidades observada e esperada foram variáveis entre as classes, sendo os maiores valores detectados nas Classes II e IV. O valor da heterozigosidade observada na Classe I $\left(\hat{H}_{o}=0,442\right)$ foi inferior a heterozigosidade esperada ( $\left.\hat{H}_{e}=0,453\right)$, mas não significativo. Na Classe II, $\hat{H}_{o}$ $(0,510)$ foi superior a $\hat{H}_{e}(0,432)$, indicando uma possível seleção em favor de heterozigotos, pois o índice de fixação $(\hat{f})$ foi negativo e significativo. Na Classe III, o valor de $\hat{H}_{o}(0,353)$ foi inferior ao de $\hat{H}_{e}(0,397)$, mostrando que a heterozigosidade observada se desvia da esperada, segundo os pressupostos do Equilíbrio Hardy-Weinberg (EHW). Na Classe IV, $\hat{H}_{o}$ foi 0,488 e $\hat{H}_{e} 0,419$, evidenciando um maior número de

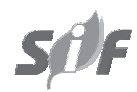

R. Árvore, Viçosa-MG, v.33, n.2, p.265-275, 2009 
heterozigotos em relação ao de homozigotos, com o índice de fixação negativo e significativo. Os desvios do EHW detectados nessas classes, em locos supostamente neutros como as aloenzimas, apontam para uma subdivisão da população, cruzamentos não aleatórios ou deriva genética (SEBBENN et al., 2003). Nas demais classes, os genótipos heterozigotos estão sendo favorecidos pelo ambiente. Esta hipótese de seleção em favor de heterozigotos é comumente relatada na literatura (BARREIRA et al., 2005; RIBAS e SEBBENN, 2004; SEBBENN et al., 2003).

O número de plântulas, árvores jovens e adultas de C. brasiliense encontrado, mostra uma redução significativa do número de indivíduos entre as classes (Tabela 1), o que pode gerar variações na distribuição da variabilidade genética nos diferentes estágios de vida. Da Classe I para a Classe II, há uma redução de aproximadamente $37 \%$, da Classe II para a III de $74 \%$ e, da Classe III para a IV de $46 \%$. Uma correlação positiva $\left(\mathrm{r}_{S}=0,80\right.$ e $\left.\mathrm{R}^{2}=0,625\right)$ foi observada entre o número de indivíduos das diferentes classes e os valores de diversidade genética, sendo que um maior o número de indivíduos proporcionará maior diversidade genética.

O valor da diversidade genética média neste estudo $(0,425)$ é próximo aos valores de outras espécies arbóreas tropicais estudadas em ambientes semelhantes. Em Copaifera langsdorffi, situada também em solos com alta saturação hídrica, a diversidade genética média foi de 0,483 (PINTO et al., 2004) e 0,396 (CARVALHO e OLIVEIRA, 2004). Sebbenn et al. (2003) encontraram para Genipa americana L., diversidade genética de 0,182 e Kawaguici e Kageyama (2001), estudando Calophyllum brasiliense, obtiveram estimativas de 0,208 , estimativas estas inferiores a encontrada nesse estudo.
A alta diversidade genética encontrada pode ser atribuída à alta densidade populacional apresentada pela espécie na área amostrada, sendo de aproximadamente 1523 indivíduos.ha ${ }^{-1}$ na Classe I, 961 na Classe II, 250 na Classe III e 135 na Classe IV. Segundo Franceschinelli et al. (2000), espécies que mantêm populações com alta densidade demográfica apresentam maiores níveis de diversidade genética do que aquelas de baixa densidade, aumentando a probabilidade de ocorrência de mutações e a incorporação de novos alelos nas populações. A mesma autora cita também que adicionalmente às mutações, a maior densidade de indivíduos pode permitir maior número de recombinações e múltiplas paternidades nas progênies, favorecendo o aumento dos níveis de diversidade genética.

\subsection{Distribuição espacial}

C. brasiliense apresentou distribuição uniforme nas quatro classes analisadas, ocorrendo nas áreas de umidade e saturação hídrica (Figura 1). A redução significativa no número de indivíduos das fases de plântula para a adulta é comum em matas. As Classes I e II, representativas das plântulas, apresentaram elevado número de indivíduos (646) e uma distribuição ampla na área, demonstrando que as condições deste ambiente são eficientes e importantes para o estabelecimento e desenvolvimento de C. brasiliens. Portanto, para garantir a perpetuação da espécie, as condições originais do ecossistema devem ser mantidas, pois intervenções nos ambientes naturais de ocorrência desta espécie poderão acarretar diminuição da variabilidade genética, essencial para a sua evolução. Como esta área encontrase em processo de degradação, será importante que medidas mitigadoras sejam efetuadas para que o desenvolvimento da espécie não seja comprometido. $\mathrm{Na}$ área observa-se entrada de gado, que pisoteia os regenerantes, portanto, medidas simples como cercar a área, já diminuiria este impacto.

Tabela 3 - Diversidade genética de plântulas, árvores jovens e adultas de Calophyllum brasiliense Camb. Â: número médio de alelos por loco; $\hat{p}$ : porcentagem de locos polimórficos; $\hat{H}_{o}$ : heterozigosidade média observada; $\hat{H}_{e}$ : heterozigosidade média esperada; e $\hat{f}$ : índice de fixação

Table 3 - Genetic diversity of seedlings, young and adult trees of Calophyllum brasiliense Camb. Â: mean number of alleles per locus; $\hat{p}$ : proportion of polymorphic loci; $\hat{H}_{o}$ : mean heterozygosity observed; $\hat{H}_{e}:$ expected mean heterozygosity and $\hat{f}$ : fixation index

\begin{tabular}{cccccc}
\hline Classes* & $\hat{A}$ & $\hat{p}$ & $\hat{H}_{o}$ & $\hat{H}_{e}$ & $\hat{f}$ \\
\hline I & 2,0 & 100 & $0,442(0,024)$ & $0,453(0,017)$ & $0,024[-0,032 ; 0,085]$ \\
II & 2,0 & 100 & $0,510(0,013)$ & $0,432(0,011)$ & $-0,181^{*}[-0,224 ;-0,143]$ \\
III & 2,0 & 100 & $0,353(0,035)$ & $0,397(0,025)$ & $0,112^{*}[0,032 ; 0,227]$ \\
IV & 2,0 & 100 & $0,488(0,043)$ & $0,419(0,022)$ & $-0,166^{*}[-0,302 ;-0,007]$ \\
\hline
\end{tabular}

* I (0,3 a 1,0 m), II (1,01 a 3,0 m), III (3,01 a 9,0 m) e IV (acima de 9,0 m).

R. Árvore, Viçosa-MG, v.33, n.2, p.265-275, 2009 

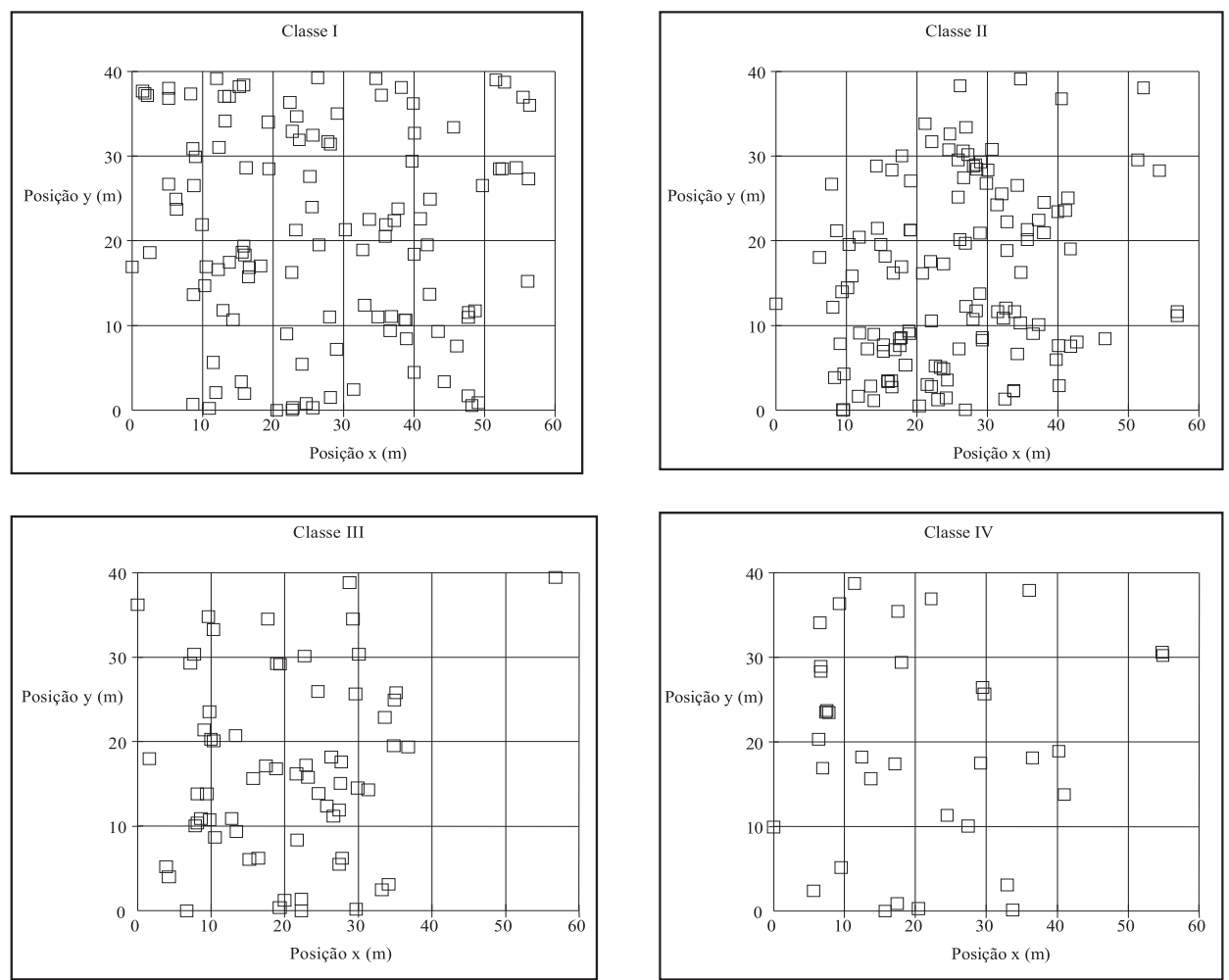

Figura 1 - Posição e distribuição espacial de plântulas, árvores jovens e adultas de Calophyllum brasiliense Camb., amostradas em um fragmento de floresta paludosa.

Figure 1 - Position and spatial distribution of seedlings, young and adult trees of Calophyllum brasiliense Camb., sampled in a swampy forest fragment.

Os correlogramas ilustrando a distribuição espacial dos genótipos de plântulas, árvores jovens e adultas de C. brasiliense são apresentados na Figura 2. No geral, a análise da distribuição espacial dos genótipos foi aleatória em todas as classes ontogênicas, para o número total de plantas e para a análise conjunta dos dados das Classes III e IV. No entanto, observase que na Classe I, as árvores mais próximas apresentaram coancestria, indicando que até $10 \mathrm{~m}$ de distância existe a probabilidade de se amostrar alelos idênticos por descendência. Isto aponta que quanto mais próximos espacialmente estão os regenerantes, maior a possibilidade de existir uma estrutura de família. Considerando que a Classe I é composta por plântulas, certamente a competição intra e interespecífica eliminará parte dos indivíduos próximos espacialmente. Na Classe III, apesar do desvio do EHW, não foi observada estrutura de famílias, portanto outros fatores como o tamanho amostral ou mortalidade das plantas devido a catástrofes ambientais, como fogo, alagamento poderiam ter causado estes desvios. As Classes III e IV apresentaram os maiores valores de intervalos de confiança, provavelmente devido ao menor número de indivíduos amostrados (Figura 2). A análise conjunta destas classes reduziu os intervalos de confiança, mas não alterou o padrão espacial aleatório observado para as classes analisadas separadamente. Este padrão aleatório de distribuição dos genótipos também foi detectado na análise conjunta de todos os genótipos.

A distribuição espacial de C. brasiliense é influenciada pelas condições edáficas do ambiente, principalmente umidade e saturação hídrica. Por isso, existe a necessidade de se conservar as áreas de sua ocorrência natural, pois a preservação e a conservação destas áreas contribuem também para a manutenção de outras espécies, que apesar de não serem consideradas especialistas em habitat, compartilham o espaço e os recursos disponíveis no local.

R. Árvore, Viçosa-MG, v.33, n.2, p.265-275, 2009 
Análise Conjunta das Classes

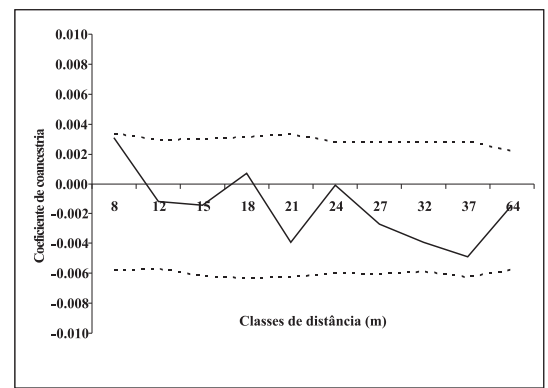

Classe II

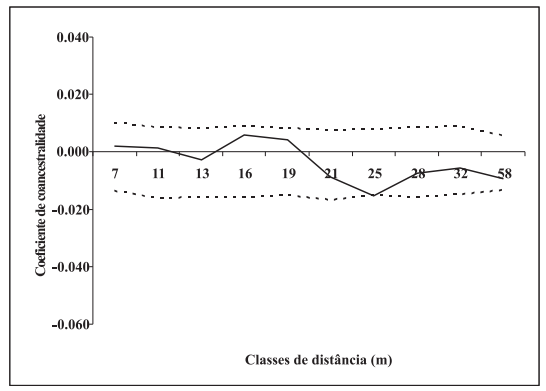

Classe IV

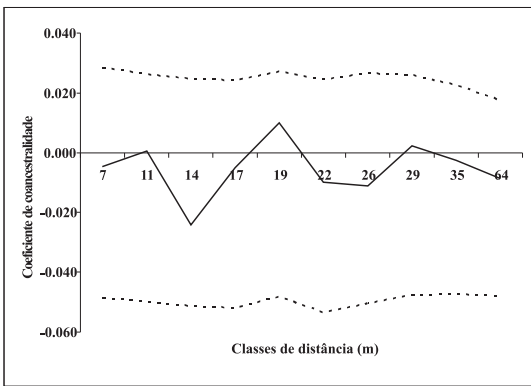

Legenda:
Classe I

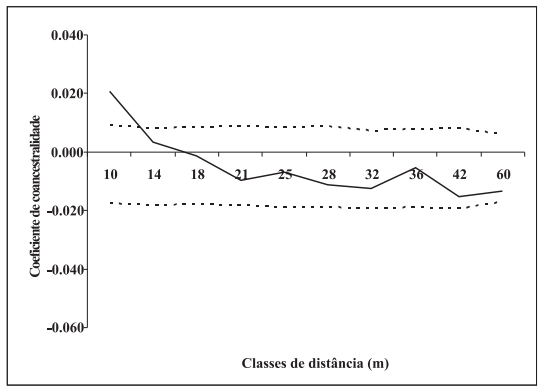

Classe III

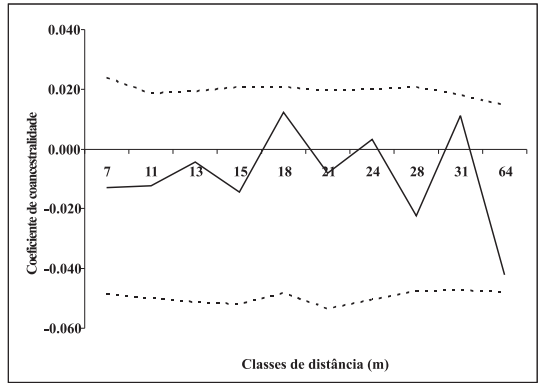

Classes III e IV

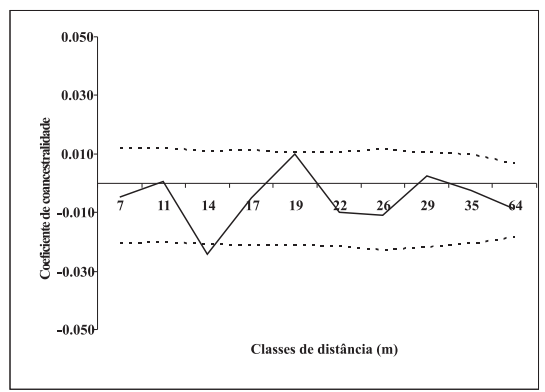

IC superior

Figura 2 - Correlogramas dos coeficientes de coancestria de plântulas, árvores jovens e adultas de Calophyllum brasiliense Camb., amostradas em um fragmento de floresta paludosa.

Figure 2 - Correlograms for the coancestry coefficients of the seedlings, young and adult trees of Calophyllum brasiliense Camb., sampled in a swampy forest fragment.

\section{CONCLUSÕES}

Foi observada alta diversidade genética entre as diferentes classes de altura e baixa variação das frequências alélicas entre as classes.

A análise da distribuição espacial dos genótipos foi aleatória na maioria das classes analisadas, com exceção dos regenerantes que apresentaram estrutura de família até $10 \mathrm{~m}$ de distância.

\section{AGRADECIMENTOS}

Ao CNPq, pela concessão da bolsa de iniciação científica à primeira autora; e a todos aqueles que contribuíram para a realização deste trabalho, em especial ao doutorando DCF/ UFLA Fábio Almeida Vieira, pela ajuda na coleta do material e pelas importantes sugestões na elaboração deste artigo.

R. Árvore, Viçosa-MG, v.33, n.2, p.265-275, 2009 


\section{REFERÊNCIAS}

ALFENAS, A. C. Eletroforese de isoenzimas e proteínas afins: fundamentos e aplicações em plantas e microrganismos. Viçosa, MG: Universidade Federal de Viçosa, 1998. 574p.

\section{ALFENAS, A. C. et al. Eletroforese de proteínas $e$ isoenzimas de fungos e essências florestais. Viçosa, MG: Universidade Federal de Viçosa, 1991. 242p.}

BARREIRA, S.; SEBBENN, A. M.; KAGEYAMA, P. Herança genética e desequilíbrio de ligação em locos isoenzimáticos de Eremanthus erythropappus. Revista do Instituto Florestal, v.17, n.2, p.183-193, 2005.

BERG, E. E.; HAMRICK, J. L. Quantification of genetic diversity at allozyme loci. Canadian Journal Forertry Research, v.27, n.3, p.415-424,1997.

BOTREL, M. C. G.; CARVALHO, D. Variabilidade isoenzimática em populações naturais de jacarandá paulista (Machaerium villosum Vog.).

Revista Brasileira de Botânica, v.27, n.4, p.621-627, 2004.

CARVALHO, D.; OLIVEIRA, A. F. Genetic structure of Copaifera langsdorffii Desf. natural populations. Cerne, v.10, n.2, p.137-153, 2004.

CARVALHO, P. E. R. Espécies florestais brasileiras: recomendações silviculturais, potencialidades e uso da madeira. Colombo: Embrapa-CNPF, 1994. 640p.

CAStro, G. C. Análise da estrutura, diversidade e florística e variações espaciais do componente arbóreo de corredores de vegetação na região do Alto Rio Grande, MG. 2004. 83f. Dissertação (Mestrado emEngenharia Florestal) Universidade Federal de Lavras, Lavras, 2004.

CHUNG, M.Y.; EPPERSON, B.K.; CHUNG, M.G. Genetic structure of age classes in Camellia japonica (Theaceae). Evolution, v57, n.1, p.62-73, 2003.

COLE, T. C. Genetic variation in rare and common plants. Annual Review of Ecology and Systematics, v.34, p.213-237, 2003
CONTE, R. et al. Genetic diversity and recruitment of the tropical palm, Euterpe edulis Mart., in a natural population from the Brazilian Atlantic Forest. Heredity, v.91, p.401-406, 2003.

FISHER, E. A. Distribuição de freqüência de classes de tamanho e estratégia reprodutiva de Calophyllum brasiliense Camb. (Guttiferae) em mata ciliar na Estação Ecológica da JuréiaItatins, São Paulo. In: SIMPÓSIO DE ECOSSISTEMAS DA COSTA SULE SUDESTE BRASIleIRA, 2., 1990, São Paulo. Anais... São Paulo: 1990. v.2. p.337-347.

FRANCESCHINELLI, E. V.; BAWA, K. S. The effect of ecological factors on the mating system of a South American shrub species (Helicteres brevispira). Heredity, v.84, n.1, p.116-123, 2000.

FRANKEL, O. H.; BROWN, A. H. D.; BURDON, J. J. The conservation of plant biodiversity. Cambridge: Cambridge University Press, 1996. p.229.

HAMRICK, J. L.; LOVELESS, M. D. The influence of seed dispersal mechanism on the genetic structure of plant populations. In: ESTRADA, A.; FLEMING, T. H. (Eds.).Frugivores and seed dispersal. Dordrecht: Dr. Junk Publications, 1986. p.211-223.

HAMRICK, J. L.; MITTON, J. B.; LINHART, Y. B. Levels of genetic variation in trees: isoenzymes of North American forest trees and forest insects. Barcklay: USDA, 1981. p.35-41. (USDA Forest Service, PSW- 48)

HARDY, O.; VEKEMANS, X. SPAGeDi 1.2: a versatile computer program to analyse spatial genetic structure at individual or population levels. Molecular Ecology Notes, v.2, p.618-620. Last update 24/03/05. Available from http://www.ulb.ac.be/sciences/lagev/software.html.

INSTITUTO BRASILEIRO DE GEOGRAFIA ESTATÍSTICA - IBGE. Mapa de vegetação do Brasil. Rio de Janeiro: 1993.

IVANAUSKAS, N. M.; RODRIGUES, R. R.; NAVES, G. Fitossociologia e seletividade de espécies numa floresta de brejo em Itatinga, SP. Revista Brasileira de Botânica, v.20, n.2, p.139-153, 1997.

R. Árvore, Viçosa-MG, v.33, n.2, p.265-275, 2009 
JAEGER, P. et al. Caracterização genética de populações naturais de Xylopia emarginata Mart. (Annonaceae). Scientia Forestalis, v.73, n.1, p.91-99, 2007.

KAWAGUICI, C. B. Germinação de sementes, estrutura e sobrevivência de uma população de Calophyllum brasiliense Camb. (Clusiaceae) em mata de galeria da Estação Ecológica do Panga. 1994. 64f. (Monografia de Graduação do Curso de Ciências Biológicas) - Universidade Federal de Uberlândia, Uberlândia, 1994.

KAWAGUICI, C. B.; KAGEYAMA, P. Y. Diversidade genética de três grupos de indivíduos (adultos, jovens e plântulas) de Calophyllum brasiliense Camb. (Clusiaceae), em uma população de mata de galeria. Scientia Forestalis, n.59, p.131-143, 2001.

LEWIS, P. O.; ZAYKIN, D. Genetic data analysis: Computer program for the analysis of allelic data. Version 1.0 (d15). Free program distributed by the authors over the internet from the GDA Home Page at http: //alleyn.eeb. uconn.edu/gda/2000.

LORENZI, H. Árvores brasileiras: manual de identificação e cultivo de plantas arbóreas do Brasil. São Paulo: Plantarum, 1992. 368p.

LOVELESS, M. D.; HAMRICK, J. L. Ecological determinants of genetic structure in plant populations. Annual Review of Ecology and Systematics, v.15, n.1, p.65-95, 1984.

MACHADO, E. L. M. et al. Análise comparativa da estrutura e flora do compartimento arbóreoarbustivo de um remanescente florestal na Fazenda Beira Lago, Lavras, MG. Revista Árvore, v.28, n.4, p.499-516, 2004.

MARQUES, M. C. M. Estudos autoecológicos do guanandi (Calophyllum brasiliense Camb. Clusiaceae) em uma mata ciliar do Município de Brotas, SP. 1994. 92f. Dissertação (Mestrado emBiologia Vegetal) - Universidade Estadual de Campinas, Campinas, 1994.

R. Árvore, Viçosa-MG, v.33, n.2, p.265-275, 2009
MORAES, M. L. T.; KAGEYAMA, P. Y.; SEBBENN, A. M. Diversidade e estrutura genética espacial em duas populações de Myracrodruon urundeuva Fr. All. Sob diferentes condições antrópicas. Revista Árvore, v.29, n.2, p.281-289, 2005.

NG, K. K. S.; LEE, S. L.; KOH, C. L. Spatial structure and genetic diversity of two tropical tree species with contrasting breeding systems and different ploidy levels. Molecular Ecology, v.13, n.3, p.657-669, 2004.

PINTO, S. I. C.; SOUZA, A. M.; CARVALHO, D. Variabilidade genética por isoenzimas em populações de Copaifera langsdorffii Desf. Em dois fragmentos de mata ciliar. Scientia Forestalis, n.65, p.40-48, 2004.

RIBAS, L. A.; SEBBENN, A. M. Herança e desequilíbrio gamético em sete locos isoenzimáticos de Cecropia pachystachya. Revista do Instituto Florestal, v.16, n.2, p.111-119, 2004.

RITLAND, K. Estimators for pairwise relatedness and individual inbreeding coefficients. Genetic Resources, v.67, n.2, p.175-185, 1996.

RUSCHELA.R.; MOERSCHBACHER, B.M.; NODARI, R.O. Demography of Sorocea bonplandii in Seasonal Deciduous Forest, Southern Brazil. Scientia Forestalis, v.70, p.149-159, 2006.

SCHIAVINI, I. Estrutura das comunidades de mata ciliar de galeria da estação Ecológica do Panga (Uberlândia, MG). Campinas, 1992. 139f. Tese (Doutorado emEcologia) - Universidade de Campinas, Campinas, 1992.

SEBBENN, A. M.; KAGEYAMA, P. Y.; VENCOVSKY, R. Conservação genética in situ e número de matrizes para a coleta de sementes em população de Genipa americana L. Scientia Forestalis, n.63, p.13-22, 2003.

SWOFORD, D. L.; SELANDER, R. B. BIOSYS-1. A fortran program for the comprehensive analysis of electrophoretic data in population genetics and systematics. Journal of Heredity, v.72, n.72, p.282-283, 1989. 
TELLES, M. P. C. et al. Caracterização genética de populações naturais de araticunzeiro (Annona crassiflora Mart. - Annonaceae) no Estado de Goiás. Revista Brasileira de Botânica, v.26, n.1, p.123-129, 2003.

TORGGLER, M. G. F.; CONTEL, E. P. B.; TORGGLER, S. P. Isoenzimas: variabilidade genética em plantas. Ribeirão Preto: SBG, 1995. 186p.

TORRES, R. B.; MATTHES, L. A. F. \& RODRIGUES, R. R. Florística e estrutura do componente arbóreo de mata de brejo em Campinas, SP. Revista Brasileira de Botânica, v.17, n.2, p.189-194, 1994.
VIEIRA, F. A.; CARVALHO, D. Genetic structure of an insect-pollinated and bird-dispersed tropical tree in vegetation fragments and corridors: implications for conservation. Biodiversity and Conservation, v.17, n.10, p.2305-2321, 2008.

VIEIRA, F. A. et al. Demographic fine-scale genetic structure of Protium spruceanum (Burseraceae), a dioecious-dominant neotropical tree, on undisturbed brazilian atlantic population (submetido).

YEEH, Y.; KANG, S. S.; CHUNG, M. G. Evaluation of the natural monument populations of Camellia japonica (Thearaceae) in Korea based on allozyme studies. Botanical Bulletin of Academia Sinica, v.37, n.1, p.141-146, 1996. 